\title{
A Structural Approach to Characterizing the Release of Outer Membrane Vesicles in Vibrio vulnificus
}

\author{
R. Guerrero-Ferreira ${ }^{1}$ and E. R. Wright ${ }^{1,2}$ \\ ${ }^{1 .}$ Emory University School of Medicine, Division of Pediatric Infectious Diseases, Children's \\ Healthcare of Atlanta, 2015 Uppergate Drive NE, Atlanta, GA 30322 \\ 2. Robert P. Apkarian Integrated Electron Microscopy Core, Emory University, 1521 Dickey Drive, \\ Atlanta, GA 30322
}

Virulence in Vibrio vulnificus is regulated by various structural components, such as cellular appendages (flagellum and pili) and the capsular polysaccharide [1]. Recently, V. vulnificus was found to produce outer membrane vesicles that can induce host cell death and may carry the cytolysin-hemolysin (VvhA), which induces cytotoxicity [2]. Here, we present cryo-electron tomography (cryo-ET) data of OMV release and distribution around the cell.

V. vulnificus clinical isolate CMCP6 was cultured overnight in LB medium $\left(250 \mathrm{rpm}\right.$ at $\left.28^{\circ} \mathrm{C}\right)$ and flash frozen onto glow-discharged Quantifoil carbon grids in liquid ethane with a FEI Vitrobot. Cryo-ET data was collected with a JEOL 2200FS $200 \mathrm{kV}$, field emission TEM with an in-column energy filter, on a Gatan 4kx4k high resolution CCD camera.

Cryo-ET data confirm our previous cryo-electron microscopy (cryo-EM) results [3], indicating that released OMVs can be found forming a series of concentric rings around the V. vulnificus cell (Fig. 1). In order to corroborate that ring formation is not an artifact of the hole's shape in Quantifoil grids; we used Lacey grids (EMS) with a support film that contains holes of various sizes and shapes. OMVs observed under these conditions also exhibited the concentric ring conformation (Fig. 2).

In addition, vesicles appear to be arranged with respect to the cell in a plane that corresponds to the position where the flagellar basal body is located (Fig. 3). Furthermore, OMVs were observed emerging from the flagellar sheath (Fig. 4), a process that, to our knowledge, has not been reported in Gramnegative bacteria. With respect to OMV content, cryo-ET revealed that cargo packaging is not a requirement for the release of OMVs. Some vesicles appeared empty, while others contained unidentified densities.

Further studies will determine the relationship between flagellar function and OMV release in $V$. vulnificus, and the contribution of environmental factors to the production of OMVs. In addition, a comparative study of the release mechanism, distribution and composition of OMVs in Vibrionaceae bacteria, may shed light onto the role they play on the ability of Vibrios to thrive in a variety of ecological niches. 


\section{References:}

[1] M.S. Strom, et al., Microbes Infect. 2(2000) 177-88.

[2] Y.R. Kim, et al., Biochem. Biophys. Res. Commun. 399(2010) 607-12.

[3] R. Guerrero-Ferreira, et al., Microscopy and Microanalysis. 17(2011) 142-143.

[4] This research was supported by funds from Emory University, Children's Healthcare of Atlanta, the Emory Center for AIDS Research, the Georgia Research Alliance, the Human Frontiers Science Program, and the National Science Foundation to E.R.W. Cryo-EM/ET data was collected at the Emory University Robert P. Apkarian Integrated Electron Microscopy Core.

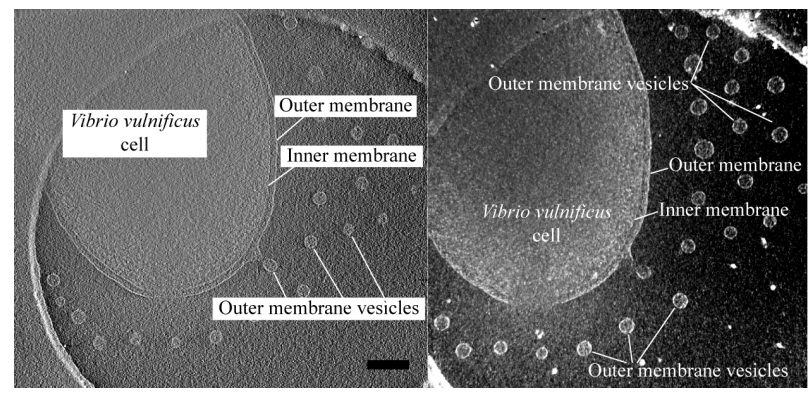

Figure 1. Averaged, 11-nm tomographic slice (left) and volume rendering (right) of a $V$. vulnificus cell illustrating the arrangement of OMVs around the cell. Sample was frozen on a Quantifoil grid. Scale bar: $200 \mathrm{~nm}$.

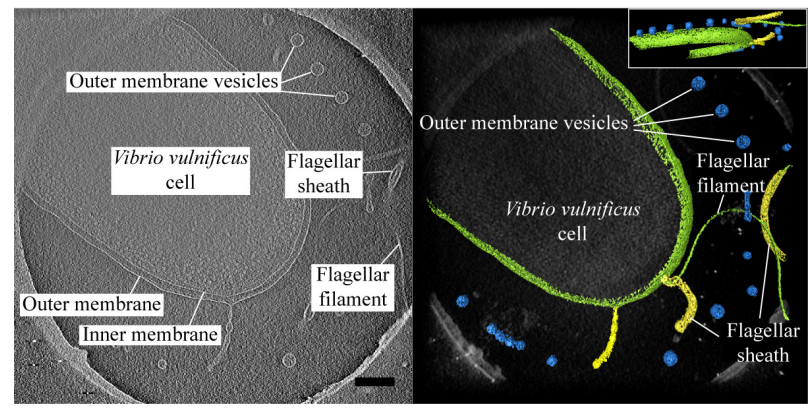

Figure 3. Averaged, 11-nm tomographic slice (left) and segmented volume imposed over rendering (right) of a $V$. vulnificus cell illustrating the arrangement of OMVs around the cell. Inset: tilted, segmented volume illustrating the plane of OMV arrangement. Scale bar: $200 \mathrm{~nm}$.

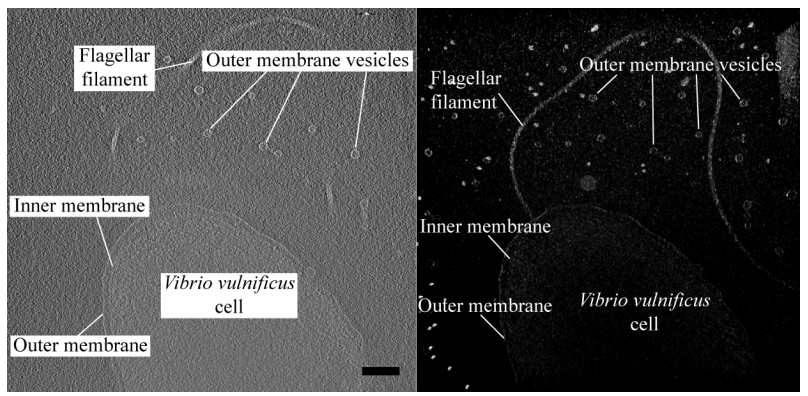

Figure 2.Averaged, 11-nm tomographic slice (left) and volume rendering (right) of a $V$. vulnificus cell depicting the concentric arrangement of OMVs around the cell. Sample was frozen on a Lacey grid. Scale bar: $200 \mathrm{~nm}$.

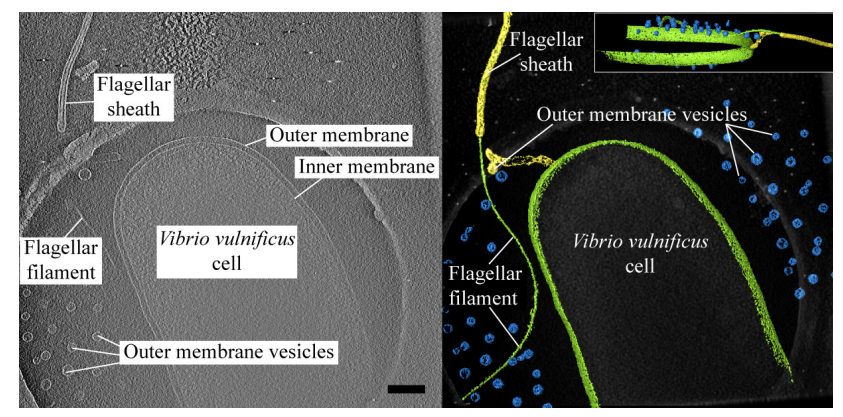

Figure 4. Averaged, 11-nm tomographic slice (left) and segmented volume imposed over rendering (right) of a $V$. vulnificus cell illustrating the arrangement of OMVs around the cell. A single OMV is observed emerging from the flagellar sheath. Inset: tilted, segmented volume illustrating the plane of OMV arrangement. Scale bar: $200 \mathrm{~nm}$. 